\title{
Anterior Interhemispheric Approach for Olfactory Groove Meningioma
}

\author{
Imam Hidayat, ${ }^{1}$ Agung Budi Sutiono, ${ }^{2}$ Ahmad Faried, ${ }^{2}$ Muhammad Zafrullah Arifin ${ }^{2}$ \\ ${ }^{1}$ Department of Neurosurgery, Faculty of Medicine, Universitas Syah Kuala-Dr. Zainal Abidin Hospital, \\ Banda Aceh, Indonesia \\ ${ }^{2}$ Department of Neurosurgery, Faculty of Medicine, Universitas Padjadjaran-Dr. Hasan Sadikin \\ General Hospital
}

$\begin{array}{ll}\text { Abstract } & \text { Objective: To evaluate the surgical technique with bifrontal interhemispheric } \\ \text { approach for total removal of tumor in olfactory groove meningioma (OGM). } \\ \text { Methods: This study described a case of a 38-year-old woman with bilateral } \\ \text { blindness, anosmia, and behaviour changes. Imaging studies show a tumor } \\ \text { mass in midfrontal base. Surgery using a bifrontal interhemispheric approach } \\ \text { was performed and total removal was achieved and postoperative computed } \\ \text { tomography (CT) scan was performed to confirm the result. Histopathological } \\ \text { findings established a diagnosis of meningioma. } \\ \text { Results: A coronal skin incision behind the hairline was utilized. The scalp } \\ \text { was elevated, taking care to reserve the vascularized pericranium medial to } \\ \text { the linea temporalis of each side, and preserving the } 2 \text { supraorbital nerves. } \\ \text { Eight burr holes were used, with the two initial holes made on each side of } \\ \text { the orbitotemporal region, and the other four holes at the midline. A bifrontal } \\ \text { craniotomy was performed. The tumor was first detached from its attachment } \\ \text { with bipolar cautery and debulked. During this step, the main tumor feeder } \\ \text { arteries from the anterior and posterior ethmoidal artery were interrupted, } \\ \text { and the tumor devascularized. Total tumor removal through surgical } \\ \text { intervention was achieved and confirmed by head CT-scan postoperatively. } \\ \text { Conclusions: This case report supports the suitability of the bifrontal } \\ \text { interhemispheric approach for OGM resection with additional radiation } \\ \text { therapy. } \\ \text { Received: }\end{array}$

\section{Introduction}

Intracranial tumors were classified by location into supra- and infra-tentorial tumors that are separated by tentorium. Supratentorial location was located on cerebral hemispheres, the lateral and third ventricles. One of the supratentorial tumors commonly found was meningioma. ${ }^{1,2}$ Olfactory groove meningioma (OGM) is a type of meningioma which is located in the midline of anterior fossa of skull base on the cribriform plane of the ethmoidal

\footnotetext{
Correspondence:

Agung Budi Sutiono, Department of Neurosurgery,

Faculty of Medicine, Universitas Padjadjaran-Dr. Hasan Sadikin General Hospital

Jl. Pasteur No. 38, Bandung, Indonesia

e-mail: agungbudis@gmail.com
}

region. ${ }^{3}$ Olfactory groove meningioma occurrs in $8-13 \%$ of all intracranial meningiomas. ${ }^{1}$ This tumor possesses several characteristics: it tends to slowly grows, usually bilateral rather than unilateral, asymmetric, and progressively compresses the frontal lobes. ${ }^{3}$ The commonly found clinical manifestations are headache, personality changes and anosmia. This tumor could extend to sellar region. If the size was big enough, it could affect the visual function by compressing the optic nerve and chiasm, thus caused visual disturbance. ${ }^{4}$

The olfactory groove meningioma is closely related to meningioma in tuberculum sella but with different clinical manifestations and surgical outcomes. Tumor in tuberculum sella could manifest early due to its relationship with the optic chiasm. ${ }^{3}$ Seizure, hemiparalysis, 


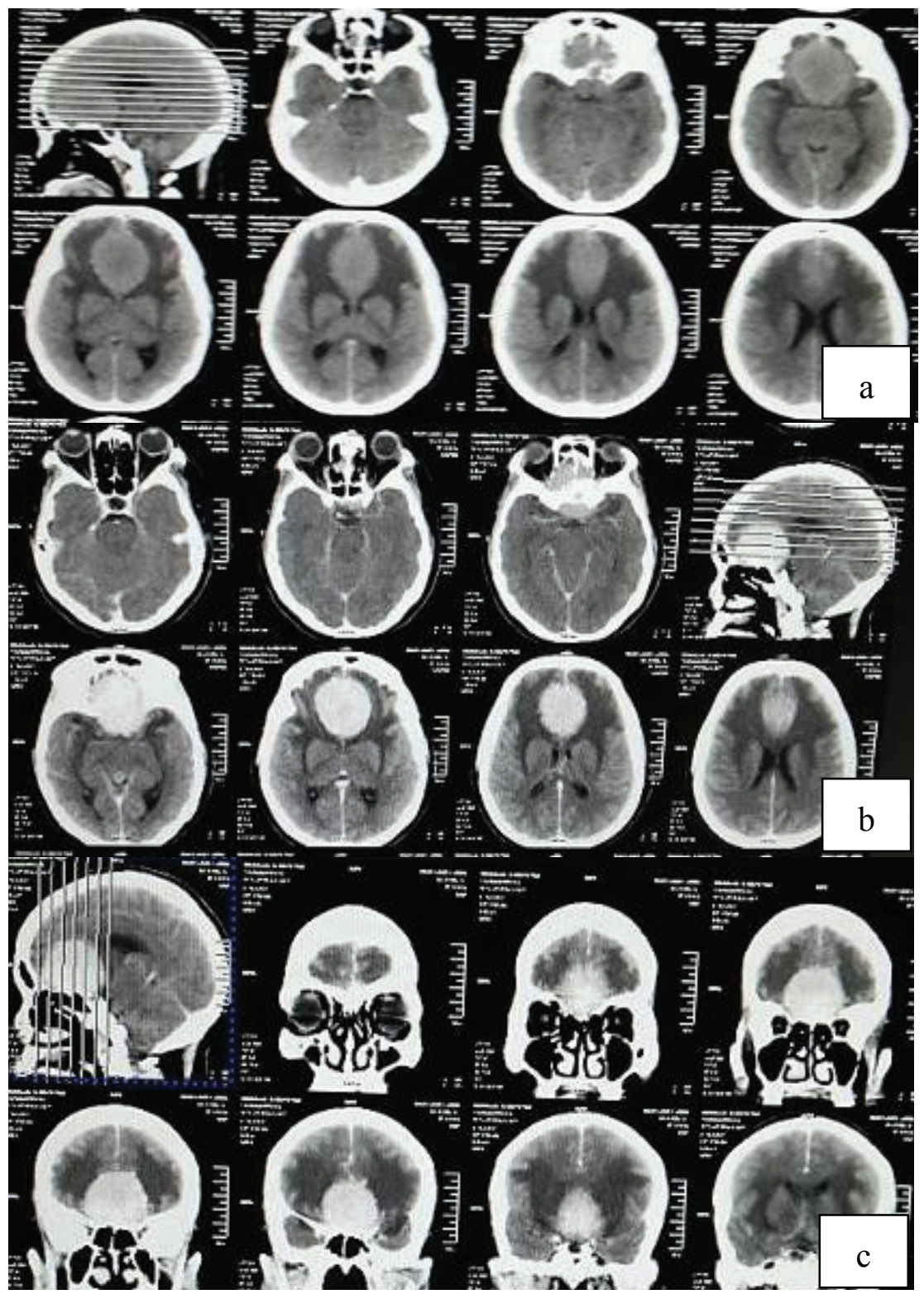

Fig. 1 Head Computed Tomography (CT) Scan at Axial Plane that Exhibited an Isodense Mass (a); Enhanced Homogenously with Contrast, at Axial Plane (b) and at Coronal Plane of Frontal Base Region (c)

and aphasia could occur in patients with OGM. Anosmia is also rarely found. ${ }^{4,5}$ The anatomical location of OGM could manifest in psychiatrical problems long before the onset of neurological deficits. Olfactory groove meningioma is one of the largest intracranial tumors detected. Brain imaging shows a meningioma in the middle subfrontal region with its extension as well as the peritumoral edema. Additionally, magnetic resonance imaging (MRI) could define the relationship between tumor and surrounding structures such as optic nerves and anterior cerebral artery and its extension to ethmoid sinuses. Surgical resection is the treatment of choice for most OGM. However, the size and the vascular and neural invasion as well as paranasal extension will increase the surgical risks. 

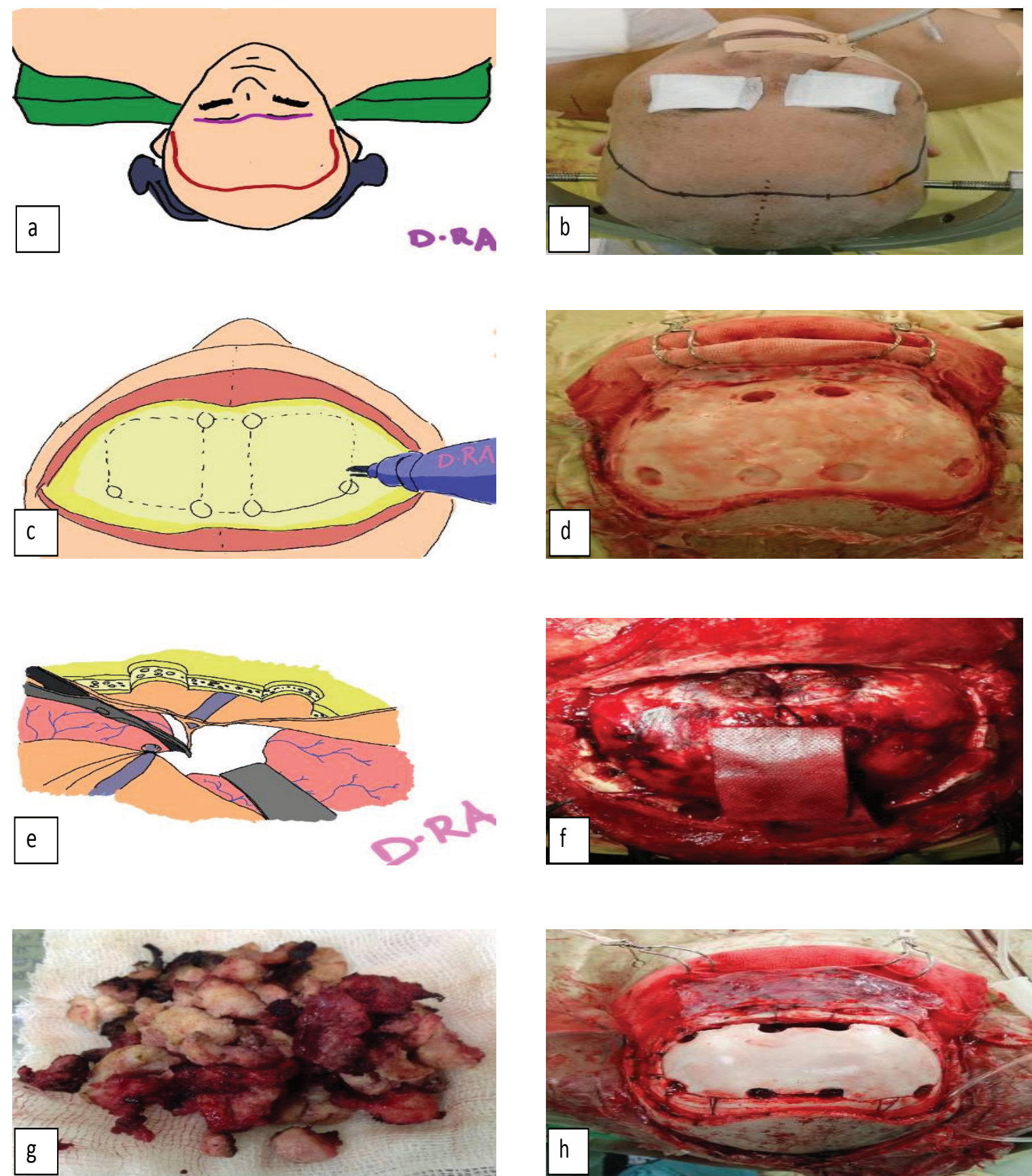

Fig. 2 Intraoperative Photograph of OGM via Anterior Interhemispheric Approach. Surgical Technique: Patient was Placed in Supine Position with Bicoronal Skin Incision (2a, b), Eight Burr Hole Bifrontal Craniotomy was Performed (2c, d). The 1/3 Anterior of Superior Sagittal Sinus was Ligated and Divided at Its Most Anterior Part (2e, f). Cut Section of Tumor was Whitish and Solid (2g). Calvaria Bone was Put Back (2h) 


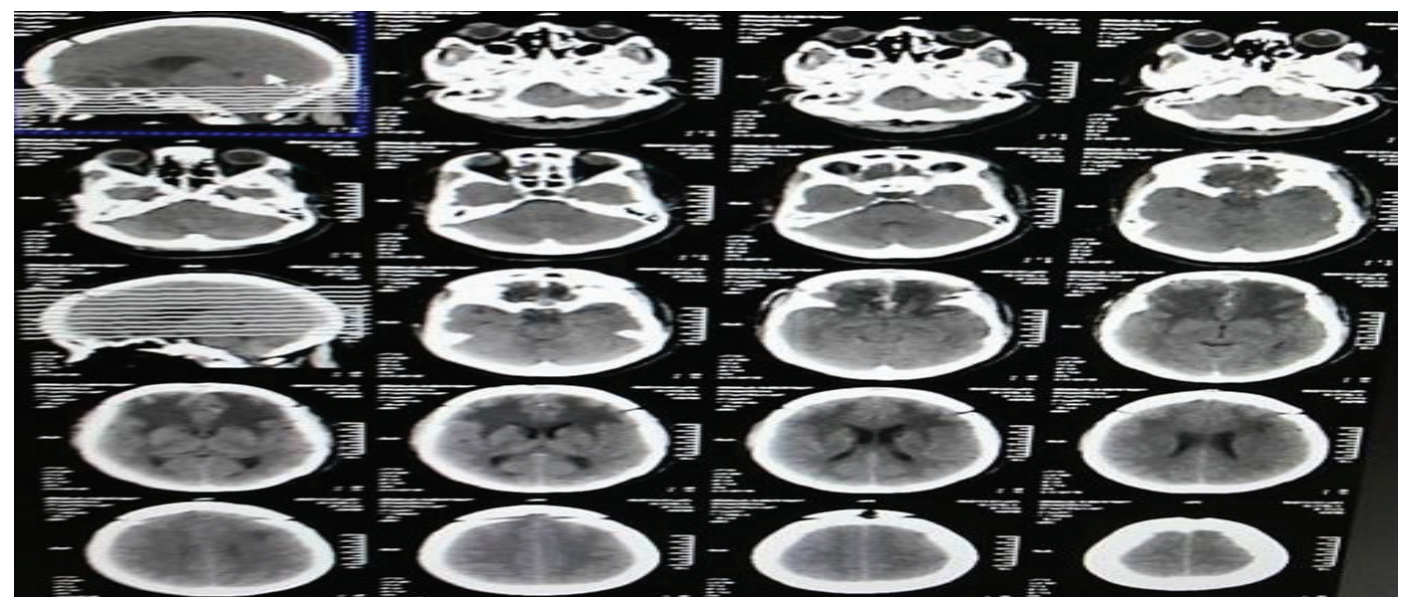

Fig. 3 Total Tumor Removal Through Surgical Intervention was Achieved and Confirmed by Head Computed Tomography (CT) Scan Postoperatively

Based on the anatomical point of view, the safest and most direct surgical access can be achieved through the tumor's anterior. Pterional and bifrontal approaches were proposed to be applied for the resection of this tumor with the preferred surgical approach, surgical tactics, and strategies depend on individual surgeon's authority. ${ }^{6-8}$ The anterior interhemispheric approach was originally developed for anterior communicating artery aneurysms. ${ }^{6}$ However, with several technical modifications, this approach yields minimum morbidity and maximizes visualization around the surrounding tuberculum sellae.

\section{Case}

A case of a 38-year-old woman who suffered from blindness of her both eyes since 3 months prior to admission is presented. The complaint was preceeded by blurred vision on both eyes since 8 months ago. The patient also experienced headache and difficulties in smelling. During those periods, the patient was also agitated and there were changes in her personality. Routine laboratory examinations showed normal findings. Brain computed tomography (CT) scan exhibited a hyperdense and homogenously enhanced mass at frontal base region (Fig. 1). A preoperative diagnosis of OGM was made. A craniotomy tumor removal was then performed (see surgical technique).
Under general anesthesia, the patient was positioned in a supine position with her back elevated about 20 degrees and the head was in a straight line above the heart to facilitate venous drainage. A coronal incision was done behind the hairline (Fig 2A and B) and the scalp was elevated by preserving the vascularized pericranium medial to the linea temporalis of each side and preserving the 2 supraorbital nerves. Eight burr holes were used, with the two initial holes made on each side of the orbitotemporal region and the other four holes were made at the midline. A bifrontal craniotomy was performed (Fig 2C and D). The medial inferior osteotomy was made close to the nasofrontal suture; therefore the frontal sinus must be opened. The frontal sinus mucosa was removed as completely as possible and was packed using tiny pieces of abdominal fat grafts soaked in fibrin glue. A viable flap of pericranium was swung over the frontal sinus and stitched to the frontal base dura at the end of the procedure. A W-shaped dura opening was made, and was reversed anteriorly. The $1 / 3$ anterior of superior sagittal sinus was ligated and divided at its most anterior part (Fig. 2E and F). The bridging veins from both frontal lobes were preseverved during this maneuver.

Tumor resection was performed according to the following steps. The tumor was first detached from its attachment with bipolar cautery and debulked. During this step, 
the main tumor feeder arteries from the anterior and posterior ethmoidal artery were interrupted, and the tumor was devascularized. The macroscopic appearance of the tumors was multiple bits of firm, irregular sized grayish white tissue. The consistency was firm to hard. Cut section was whitish and solid (Fig. 2G); calvaria bone was put back (Fig. 2H). The final histopathological result indicated meningioma. Total tumor removal through surgical intervention was achieved and then confirmed by head CT-scan postoperatively (Fig. 3).

\section{Discussion}

Olfactory groove meningioma (OGM) from midline anterior skull base is rare; it represents $10 \%$ of all intracranial meningiomas. This tumor usually arises from ethmoid, planum sphenoid or frontosphenoid region; the weakest part of skull base, which makes it prone to bone infiltration. The tumor could also extend to paranasal sinuses and nasal cavity. This tumor could also suppress the olfactory nerve at lateral and posterior part of optic chiasm. This tumor usually attached to the duramater. This tumor receives vascularization from anterior and posterior ethmoidal artery. ${ }^{4}$ Histological features could be used for the classification of meningioma and predicting its recurrence rate and survival rate. About $90 \%$ of grade I OGM was benign with recurrence rate after surgical resection between 7-20\%. ${ }^{1}$

Olfactory groove meningioma is a slowgrowing tumor with defined borders which enables it to grow in size before it becomes symptomatic. Symptoms are usually manifest if there are compressions on the brain tissue. If the tumor is accessible, the surgery is performed to remove the tumor and will involve structures such as duramater and bones. The extent of tumor removal is important for longterm tumor control. Evaluation for feeding arteries is important because in several cases, tumor embolizations are performed to facilitate tumor removal. Radiation therapy is performed in cases where surgical option is not available. Sometimes, for asymptomatic patients, surgery is optional. The incidence of atypical grade II tumors is 5-7\% with recurrence rate about $40 \%$. It is not clear whether this grade was considered benign or malignant. But if there is a brain invasion, there are tendencies of faster growth and recurrence. ${ }^{1,4}$ Radiation therapy is especially considered after surgical intervention if there are tumor residues. Less than 3\% of cases are classified as grade III, which are malignant or anaplastic with $50-80 \%$ recurrence rate. ${ }^{7}$

Compression on the frontal lobes could manifest in cognitive and behavioral changes. The most common symptoms are headache, visual impairment, and anosmia which may create bias with other diseases such as sinusitis, migraine, and neuralgia. In a retrospective study on 59 patients with meningioma, the preoperative assessment revealed that the most common symptoms include dizziness, seizure, mental disturbances, headache, visual impairment, papilledema and hemiparalysis. ${ }^{3}$ Regarding the tumor location, symptoms such as attention deficits and emotional detachment could be early signs for tumor growth. Anosmia is rarely found as an initial finding. Long-term psychiatric manifestation could manifest before the neurological deficits are apparent. Due to its subtle manifestation, OGM could grow in a comparable size before becoming clearly symptomatic.

Tumor sizes were categorized using the neuroimaging evaluation. A tumor can be classified as small $(0-2 \mathrm{~cm}$ in diameter), medium (2-4 cm in diameter), large (4-6 cm in diameter) and giant ( $>6 \mathrm{~cm}$ in diameter). Recent study on 59 OGM patients show that the tumor size was large in 32 cases $(54.2 \%)$ and giant in 11 cases $(18.7 \%)$. Visual impairment usually occurs after tumor grows in comparable sizes. Due to the posterior extension, OGM could compress one or both optic nerves, or even optic chiasm. Surgical approaches available for OGM are pterional, unilateral and subfrontal bifrontal. ${ }^{3}$ The extents of the surgical resection in OGM are based on Simpson grading, which was found by Simpson on 1957. OGM resection is divided into 5 categories: grade I which is a complete tumor removal involving dural and vein resection, grade II which is a visible tumor resection with coagulation of dural attachment, grade III which is an intradural tumor resection without coagulation of dural attachment or extradural extension, grade IV which is a partial resection with intradural tumor left in situ, and grade $\mathrm{V}$ which is conducting decompression with or without biopsy. ${ }^{5}$

There are many publications of surgical approaches to OGM since it was first reported. All of these approaches have their advantages and disadvantages, but the most commonly used approaches are the pterional and bifrontal approaches along with their modifications. ${ }^{8}$ The anterior interhemispheric dissection is 
classically used to expose a variety of midline pathological lesions involving the suprasellar cistern, such as craniopharyngiomas ${ }^{9}$ and anterior communicating artery aneurysms. ${ }^{2}$ The interhemispheric approach has rarely been reported for the use of removing midline meningiomas. ${ }^{10}$ Our rationale in using this approach is to take advantage of the natural anatomical terrain to expose the base of the brain without significant retraction. Most authors agree that preserving vision is achieved by minimizing direct trauma to the optic nerve and avoiding injury to the

\section{References}

1. Kaprealian T, Raleigh DR, Sneed PK, Nabavizadeh N, Nakamura JL, McDermott MW. Parameters influencing local control of meningiomas treated with radiosurgery. J Neurooncol. 2016;128(2):357-64.

2. Bang $M$, Suh JH, Park JB, Weon YC. Pure Intrasellar meningioma mimicking pituitary macroadenoma: MR imaging and review of the literature. World Neurosurg. 2016;91(6): 675-9.

3. Ciurea AV, Iencean SM, Rizea RE, Brehar FM. Olfactory groove meningiomas: a retrospective study on 59 surgical cases. Neurosurg Rev. 2012;35(2):195-202.

4. Fox D, Khurana VG, Spetzler RF. Olfactory groove/planum sphenoidale meningiomas. In Lee JH, editor: Meningiomas. New York: Springer, 2009 p. 327-32.

5. Le Rhun E, Taillibert S, Chamberlain MC. Systemic therapy for recurrent meningioma. Expert Rev Neurother. 2016;16(8):889-901.

6. Nimmannitya P, Goto T, Terakawa Y, Sato H, vascular supply of the optic apparatus. The key to success for surgical excision of the OGM is by maintaining the arachnoid plane. Therefore, the best surgical approach requires the followings: improved visualization of the tumor and the surrounding vital structures, and a bloodless surgical field.

In conclusion, a case of OGM in a 38-yearsold woman and the use of surgery and aggressive adjuvant treatment, such as radiation therapy, through the use of the anterior interhemispheric approach has been reported.
Kawashima T, Morisako $\mathrm{H}$, et al. Characteristic of optic canal invasion in 31 consecutive cases with tuberculum sellae meningioma. Neurosurg Rev. 2016 Apr 27. [serial on the internet]. 2016 Apr [cited 2016 may 2];39(146):[about 7p.]. Available from: http://link.springer.com/artic le/10.1007\%2Fs10143-016-0735-6.

7. Norden AD, Drappatz J, Wen PY. Advances in meningioma therapy. Curr Neurol Neurosci Rep. 2009;9(3): 231-40.

8. Samii M, Gerganov VM. Surgery of extra-axial tumors of the cerebral base. Neurosurgery. 2008;62(6 Suppl 3):1153-61.

9. Nussbaum-Hermassi L, Ahle G, Zaenker C, Duca C, Namer IJ. Optic nerve sheath meningioma detected by single- photon emission computed tomography/computed tomography somatostatin receptor scintigraphy: a case report. J Med Case Rep. 2016;10(1):96-100.

10. Terasaka S, Asaoka K, Kobayashi H, Yamaguchi S. Anterior interhemispheric approach for tuberculum sellae meningioma. Neurosurgery. 2011;68(1 Suppl):84-9. 